\title{
L-glutamine provides acid resistance for Escherichia coli through enzymatic release of ammonia
}

\author{
Peilong $\mathrm{Lu}^{1,2,3,{ }^{*}}$, Dan $\mathrm{Ma}^{1,2,3,{ }^{*}}$, Yuling Chen ${ }^{1,2}$, Yingying Guo ${ }^{2,4}$, Guo-Qiang Chen ${ }^{2,4}$, Haiteng Deng ${ }^{1,2}$, \\ Yigong $\mathrm{Shi}^{1,2,3}$
}

${ }^{I}$ Ministry of Education Key Laboratory of Protein Science, School of Life Sciences and School of Medicine, Tsinghua University, Beijing 100084, China; ${ }^{2}$ Tsinghua-Peking Joint Center for Life Sciences, School of Life Sciences and School of Medicine, Tsinghua University, Beijing 100084, China; ${ }^{3}$ Center for Structural Biology, School of Life Sciences and School of Medicine, Tsinghua University, Beijing 100084, China; ${ }^{4}$ Ministry of Education Key Laboratory of Bioinformatics, School of Life Sciences and School of Medicine, Tsinghua University, Beijing 100084, China

Bacteria, exemplified by enteropathogenic Escherichia coli $(E$. coli), rely on elaborate acid resistance systems to survive acidic environment (such as the stomach). Comprehensive understanding of bacterial acid resistance is important for prevention and clinical treatment. In this study, we report a previously uncharacterized type of acid resistance system in $E$. coli that relies on L-glutamine (GIn), one of the most abundant food-borne free amino acids. Upon uptake into $E$. coli, Gln is converted to L-glutamate (Glu) by the acid-activated glutaminase YbaS, with concomitant release of gaseous ammonia. The free ammonia neutralizes proton, resulting in elevated intracellular $\mathrm{pH}$ under acidic environment. We show that YbaS and the amino acid antiporter GadC, which exchanges extracellular Gln with intracellular Glu, together constitute an acid resistance system that is sufficient for $E$. coli survival under extremely acidic environment.

Keywords: acid resistance; bacterial stress response; glutaminase; glutamine; amino acid antiporter Cell Research (2013) 23:635-644. doi:10.1038/cr.2013.13; published online 22 January 2013

\section{Introduction}

The food-borne enterohemorrhagic bacteria pose a global health threat. The Escherichia coli (E. coli) strain O157:H7 caused a worldwide pandemic in 1982 [1]; whereas the strain O104:H4 was responsible for the European outbreak in 2011 [2], killing 18 and sickening more than 1500 people. Both to pass through the stomach ( $\mathrm{pH} 2)$ and to survive in other acidic environment, E. coli has developed sophisticated acid resistance systems (ARs) [3]. Of the three known ARs, the functional mechanism of AR1 remains elusive; it requires $\sigma^{\mathrm{S}}$ and CRP-activated genes, and can be suppressed by glucose [4-7]. By contrast, the molecular mechanisms of AR2 and AR3 have been convincingly deciphered.

\footnotetext{
*These two authors contributed equally to this work. Correspondence: Yigong Shi

E-mail: shi-lab@tsinghua.edu.cn

Received 4 December 2012; revised 12 December 2012; accepted 13 December 2012; published online 22 January 2013
}

AR2 comprises an amino acid antiporter GadC [8, 9], which exchanges extracellular L-glutamate (Glu) with intracellular $\gamma$-aminobutyric acid (GABA), and two Glu decarboxylases GadA and GadB [10, 11], which convert Glu to GABA. Similar to AR2, AR3 also has two components, an L-arginine (Arg)-agmatine antiporter AdiC $[12,13]$ and an Arg decarboxylase AdiA [14]. The net consequence of each complete cycle by AR2 or AR3 is expelling of a cytoplasmic proton to the extracellular environment, thus increasing intracellular $\mathrm{pH}$ and consequently promoting survival under acidic environment.

Comprehensive and mechanistic knowledge on bacterial AR is a prerequisite for effective therapeutic intervention. As all enteropathogenic bacteria, mostly foodborne, must pass through the extremely acidic stomach, mechanisms of bacterial survival under environmental $\mathrm{pH}$ values of 2-3 are of pivotal significance. At present, our understanding of these mechanisms is far from complete.

GadC was recently found to efficiently transport not only Glu and GABA, but also Gln and L-methionine, in a proteoliposome-based in vitro assay [15]. For example, 
the $V_{\max }$ and $K_{\mathrm{m}}$ values for Gln are comparable to, or even better than, those for Glu [15]. This finding, together with the fact that Gln is an extremely abundant free amino acid in a large variety of food sources (Supplementary information, Table S1), motivated us to investigate whether Gln can be exployed by bacteria such as E. coli to promote survival under acidic environment. In this study, we report the discovery of a new acid resistance system in E. coli, which relies on the glutaminase $\mathrm{YbaS}$ and the membrane transporter GadC. This finding is important for understanding bacterial physiology and may offer insights into the antibiotic design for enteropathogenic bacteria.

\section{Results}

\section{Acid resistance by L-glutamine}

Glu and Arg are known to help bacteria survive the acidic environment, through the actions of AR2 and AR3, respectively [3]. To systematically examine the roles of other amino acids, we employed a bacterial survival assay [7] (Figure 1A). E. coli culture was started in buffered LB medium supplemented with $0.4 \%$ glucose, which would suppress AR1, and allowed to grow overnight to reach stationary phase. Then, an aliquot of the culture was diluted 100-fold into the EG medium, $\mathrm{pH} 2.5$, supplemented with one of the 20 natural L-amino acids. After $2 \mathrm{~h}$, cells were checked for viability on LB agar plates.

This result was unequivocal - only two amino acids, Glu and Gln, markedly enhanced the survival of $E$. coli at pH 2.5 under these experimental conditions (Figure 1B). The survival percentages of $E$. coli were similar for Glu and Gln. Notably, neither Arg nor any other amino acid provided significant acid resistance in this experimental system. The observation that Arg failed to confer acid resistance is consistent with the knowledge that the Arg decarboxylase AdiA is insufficiently induced in the LB medium [16]. These results unambiguously demonstrate that Gln alone is sufficient for acid resistance in E. coli.

To examine whether Gln-mediated acid resistance is unique to E. coli, we performed a similar set of experiments in another enteropathogenic bacteria Shigella flexneri (S. flexneri). Consistent with the results of E. coli, survival of $S$. flexneri under acid shock of $\mathrm{pH} 2.5$ was markedly enhanced by the presence of Gln and Glu, but not by any other amino acid (Supplementary information, Figure S1). These results suggest that Gln might be generally important for acid resistance in bacteria.

Requirement of the glutaminase YbaS for acid resistance

There are at least two potential mechanisms by which

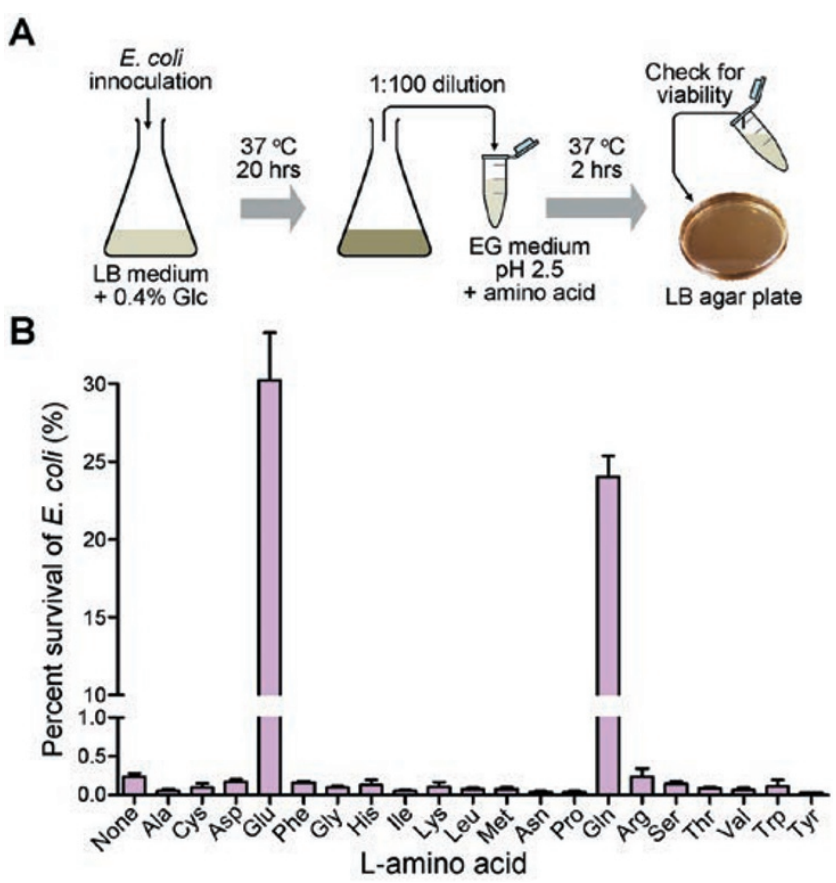

Figure $1 \mathrm{GIn}$ markedly enhances survival of $E$. coli under acid shock of $\mathrm{pH} 2.5$. (A) A schematic diagram of an acid resistance assay. Upon inoculation into buffered LB medium supplemented with $0.4 \%$ glucose (to suppress AR1), E. coli was grown overnight to reach stationary phase. The culture was diluted 1:100 into EG medium, $\mathrm{pH} 2.5$, supplemented with a specific amino acid. $2 \mathrm{~h}$ later, cells were checked for viability on LB agar plates. (B) Only Glu and GIn provide strong resistance to the acid shock. Approximately $30 \%$ and $25 \%$ E. coli survived with Glu and GIn as the amino acid supplement, respectively. Survival percentage was below 0.5 for any other amino acid. The results shown are the average of three independent experiments, with the SDs indicated.

Gln may provide acid resistance. One involves decarboxylation, similar to that for Glu by GadA/GadB; and the other involves enzymatic deamidation. Rigorous search of the NCBI database failed to identify any specific Gln decarboxylase in E. coli. In addition, although recombinant GadA or GadB protein efficiently decarboxylates Glu to generate GABA (Supplementary information, Figure S2A-S2F), neither can use Gln as a substrate (Supplementary information, Figure $\mathrm{S} 2 \mathrm{G}$ and $\mathrm{S} 2 \mathrm{H}$ ). These observations effectively rule out decarboxylation as the primary mechanism for Gln-mediated acid resistance. The other possibility - enzymatic deamidation - requires glutaminase. Much to our delight, E. coli contains two glutaminase genes, $y b a S$ and $y n e H$, which are thought to function at acidic and neutral $\mathrm{pH}$ ranges, respectively [17-19] (Figure 2A). We hypothesized that ybaS might be essential for conferring Gln-mediated acid resistance 
in E. coli.

To examine this hypothesis, we generated two E. coli variants, one with the $y b a S$ gene deleted $(\Delta y b a S)$ and the other with $y n e H$ removed $(\Delta y n e H)$, and compared their survival to the wild-type (WT) E. coli under acidic environment of $\mathrm{pH} 2.5$. As anticipated, survival of the WT E. coli was greatly facilitated by the presence of either $1 \mathrm{mM}$ Glu or $1 \mathrm{mM}$ Gln (Figure 2B). The variant $\Delta y n e H$ behaved similarly as the WT E. coli (Figure 2B), suggesting that the glutaminase YneH may not play an important role in acid resistance. In sharp contrast to the WT E. coli and the variant $\Delta y n e H$, survival of the $\Delta y b a S$ variant was only enhanced by the presence of $1 \mathrm{mM} \mathrm{Glu}$, but not by $1 \mathrm{mM}$ Gln (Figure 2B). These experimental results demonstrate that ablation of the $y b a S$ gene abolishes Gln-mediated acid resistance in $E$. coli, identifying YbaS as the essential glutaminase for acid resistance.

Next, we attempted to rescue survival of the E. coli variant $\triangle y b a S$ under acidic condition through provision of an exogenous plasmid that expresses the $y b a S$ gene. Under acidic conditions where these $E$. coli failed to survive in the presence of $1 \mathrm{mM} \mathrm{Gln}$, transformation of the $y b a S$-containing plasmid, but not the empty plasmid, allowed robust survival (Figure 2C). Expression of the YbaS protein was detected only in cells transformed with the $y b a S$-containing plasmid, but not with the empty plasmid (Supplementary information, Figure S3, lanes 1 and 2). Together, our experimental evidence demonstrates that the glutaminase $\mathrm{YbaS}$ is required for acid resistance in E. coli.

\section{Activation of YbaS at acidic $\mathrm{pH}$}

To confirm our genetic observations, we overexpressed $y b a S$ and $y n e H$ in $E$. coli, and biochemically purified the two recombinant proteins to homogeneity (Supplementary information, Figure S4). The glutaminase activities of $\mathrm{YbaS}$ and $\mathrm{YneH}$ were determined in the $\mathrm{pH}$ range of 4.0-8.0 (Figure 2D). Strikingly, YbaS was activated by acidic $\mathrm{pH}$ and exhibited robust glutaminase activity only at $\mathrm{pH} 6.0$ or lower. The glutaminase activity of $\mathrm{YbaS}$ was the highest at $\mathrm{pH} 4.0$, followed by $\mathrm{pH} 5.0$ and 6.0. At a substrate concentration of $3.3 \mathrm{mM} \mathrm{Gln}$, the glutaminase activity of $\mathrm{YbaS}$ at $\mathrm{pH} 6.6$ was $\sim 3 \%$ of that at $\mathrm{pH} 6.0$. At the $\mathrm{pH} 7.0$ and 8.0, YbaS had no detectable activity (Figure 2D). By sharp contrast, YneH displayed high levels of glutaminase activity only at $\mathrm{pH} 6.0$ or higher, with undetectable activity at pH 5.0 or lower (Figure 2D).

The reciprocal glutaminase activities of $\mathrm{YbaS}$ and YneH with respect to $\mathrm{pH}$ values were further confirmed by their maximal activities $\left(V_{\max }\right)$ (Figure 2E). The $V_{\max }$ values of $\mathrm{YbaS}$ at $\mathrm{pH} 4.0$ and 5.0 are similar to those of $\mathrm{YneH}$ at $\mathrm{pH} 8.0$ and 7.0, respectively. Compared to these $V_{\max }$ values, both $\mathrm{YbaS}$ and $\mathrm{YneH}$ displayed a reduced level of catalytic activity at $\mathrm{pH} 6.0$ (Figure 2E). The measured glutaminase activities for $\mathrm{YbaS}$ and $\mathrm{YneH}$ are generally consistent with those reported for E. coli glutaminase A and B, respectively [18, 19]. Thus YbaS and YneH likely correspond to glutaminase A and B, respectively. Together with published reports, our experimental evidence provide a solid biochemical basis for the conclusion that the glutaminase $\mathrm{YbaS}$, but not $\mathrm{YneH}$, is required for Gln-mediated acid resistance in E. coli.

\section{Enzymatic release of ammonium by $Y$ baS}

The catalytic activity of glutaminase YbaS should result in generation of the gaseous ammonia. We speculated that some ammonia might be released into the culture medium, perhaps through the action of ammonia transporter or simple membrane permeation [20]. Under

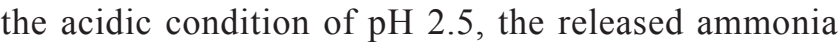
should be rapidly protonated into ammonium ion, which has a pKa of 9.25. In this case, we might be able to detect the accumulation of ammonium ion. To examine this scenario, we designed an in vitro assay to continuously measure ammonium concentration (Figure $3 \mathrm{~A}$ ). In this assay, E. coli cells are resuspended in $25 \mathrm{mM}$ citrate buf-

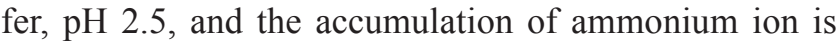
monitored by a specialized ammonium meter.

Supporting our conjecture, the concentration of ammonium ion increased steadily with time for the WT $E$. coli and the $\Delta y n e H$ variant in the presence of $1 \mathrm{mM}$ Gln (Figure 3B). By contrast, ammonium concentration remained at the basal level irrespective of time for the $E$. coli variant $\triangle y b a S$ (Figure 3B), confirming loss of the glutaminase activity at acidic $\mathrm{pH}$. The temporal increase of ammonium concentration was strictly dependent on the presence of Gln, but not Asn or Glu (Figure 3C; Supplementary information, Figure S5A). In addition, compared to that of $1 \mathrm{mM}$ Gln, the rate of increase of ammonium concentration in the buffer was enhanced by $\sim 5$-fold in the presence of $10 \mathrm{mM}$ Gln (Figure 3B and 3D). Finally, supporting the conclusion of the $y b a S$ rescue experiment (Figure 2C), provision of the $y b a S$ expression plasmid, but not the empty plasmid, allowed the E. coli variant $\triangle y b a S$ to release ammonium (Supplementary information, Figure S5B). Together, these results show that the released ammonium is derived from enzymatic activity of the glutaminase YbaS.

\section{Requirement of GadC}

For YbaS to mediate acid resistance in E. coli, Gln in the culture medium must be loaded into the cells. Given its known transport activity for Gln [15], the amino acid antiporter GadC is likely responsible for the uptake of 
A

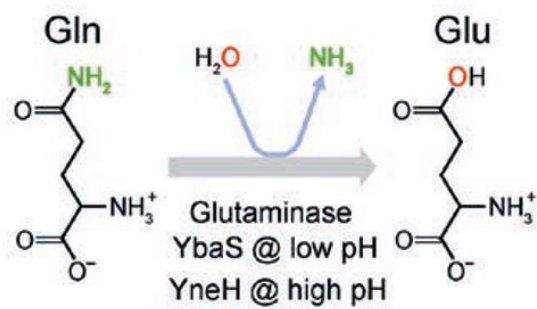

C

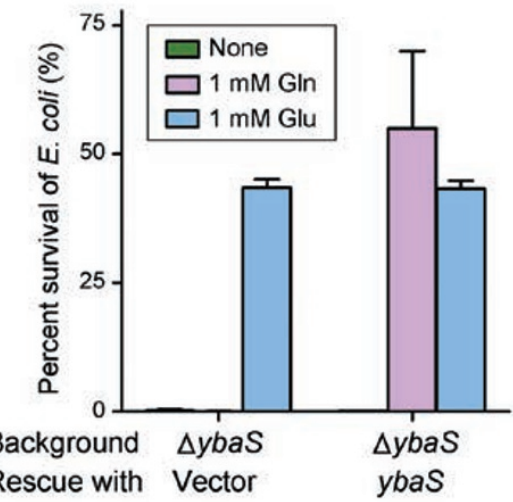

B

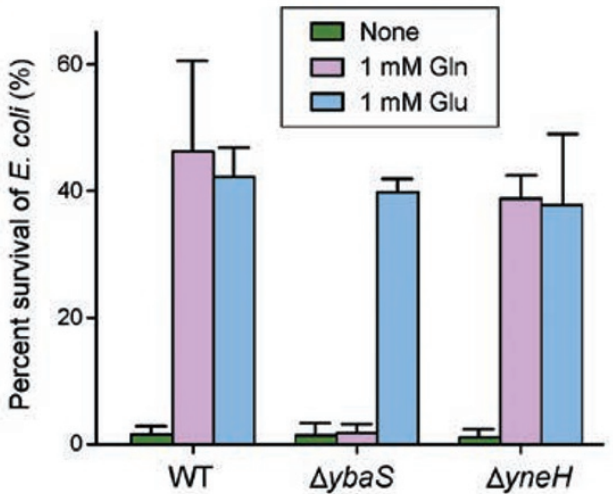

E

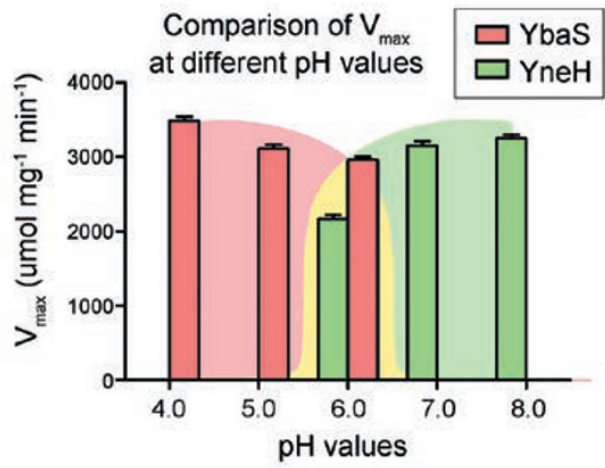

D
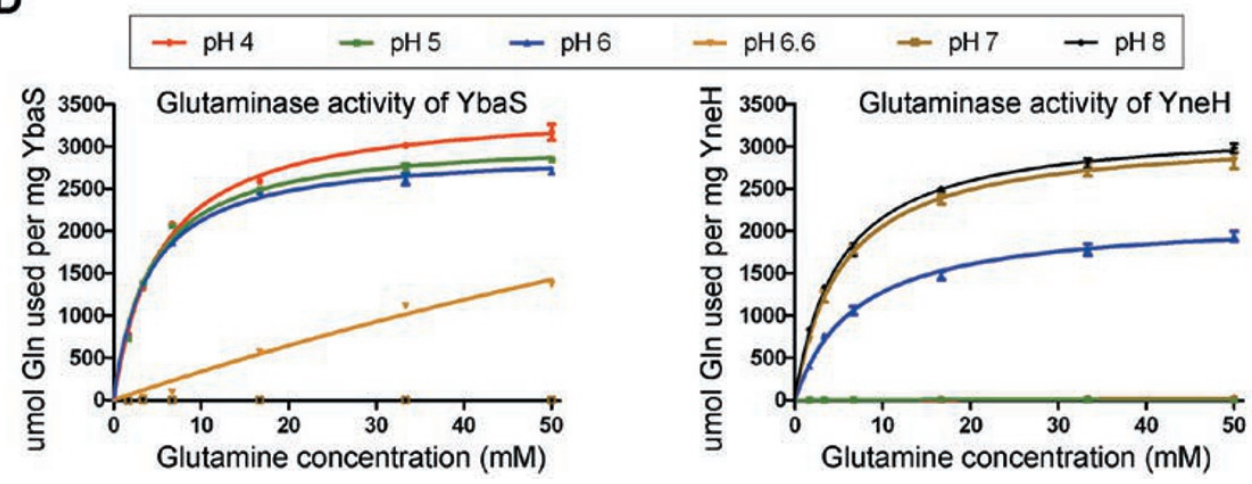

Figure 2 The glutaminase YbaS is required for Gln-mediated acid resistance in E. coli. (A) The reaction catalyzed by glutaminase results in the release of a molecule of gaseous ammonia. E. coli has two glutaminases, YbaS and YneH, which are thought to function at acidic and neutral/basic $\mathrm{pH}$ ranges, respectively. (B) The $E$. coli glutaminase gene $y b a S$ is required for GIn-mediated acid resistance. Deletion of the glutaminase gene ybaS led to abrogation of acid resistance in the presence of $1 \mathrm{mM}$ GIn. By contrast, deletion of $y n e H$ had no effect on acid resistance. (C) GIn-dependent survival of $E$. coli $\Delta y b a S$ under acidic environment can be rescued by transformation of a ybaS-expressing plasmid. (D) YbaS exhibits robust glutaminase activity only at $\mathrm{pH} 6$ or lower. By contrast, the glutaminase YneH displays activity only at pH 6 or higher. (E) A summary of the $\mathrm{pH}$-dependent $V_{\max }$ values of YbaS and $\mathrm{YneH}$. The results shown in panels (B-E) are the average of three independent experiments, with the SDs indicated.

Gln from the medium and the release of reaction products into the medium. We hypothesized that, under these circumstances, GadC might be required for Gln-mediated acid resistance. To examine this hypothesis, we generated an E. coli variant with the deletion of $\operatorname{gadC}(\triangle \mathrm{gadC})$ and compared its survival to the WT E. coli under the acidic environment of $\mathrm{pH} 2.5$. Loss of the gadC gene not only crippled Glu-mediated acid resistance but also completely abolished the ability of $E$. coli to resist acidic shock in the presence of Gln (Figure 4A), confirming 


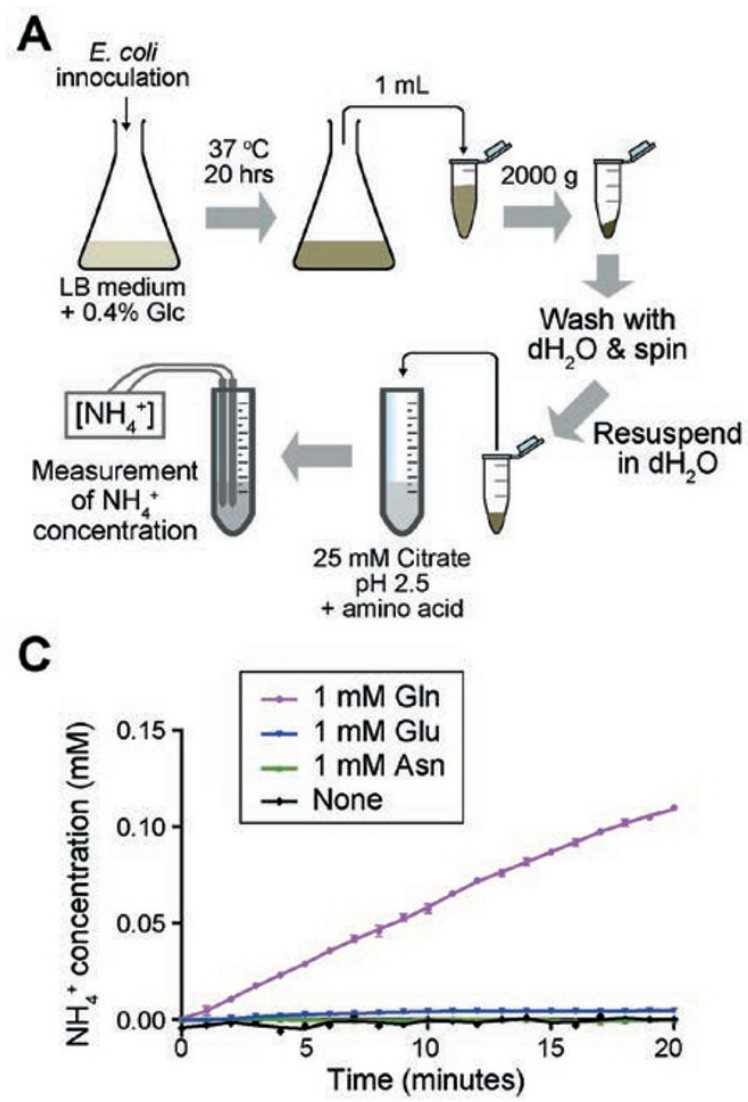

B

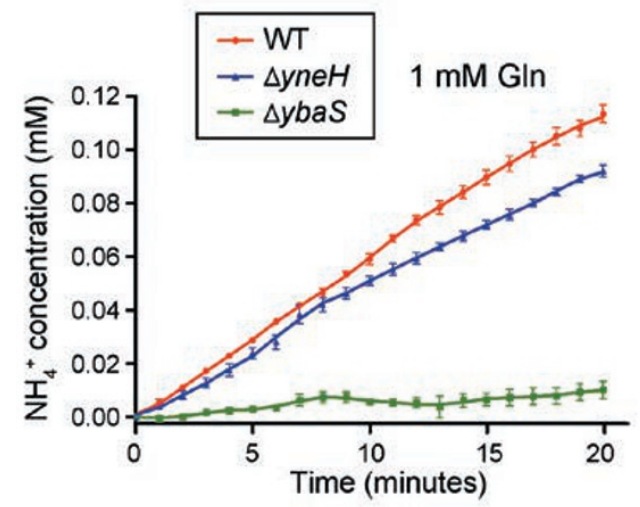

D

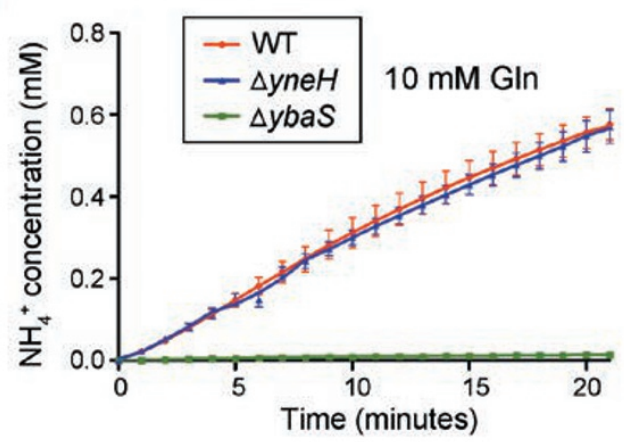

Figure 3 Ammonium is released through the action of YbaS. (A) A schematic diagram of the ammonium measurement assay. Upon inoculation into LB medium supplemented with $0.4 \%$ glucose (to suppress AR1), E. coli was grown overnight to reach stationary phase. The cells were pelleted by centrifugation, washed with distilled water, pelleted again and resuspended in distilled water before mixing with $10 \mathrm{ml}$ of citrate buffer, $\mathrm{pH} 2.5$, supplemented with a specific amino acid. The ammonium concentration was continuously measured by a special meter. (B) Deletion of the ybaS gene resulted in no increase of ammonium concentration in the buffer. By contrast, ammonium concentration in the buffer increased steadily over time for WT or $\Delta y n e H$ E. coli. $1 \mathrm{mM}$ Gln was present in the buffer. (C) Ammonium concentration steadily increased only in the presence of GIn, but not Glu or Asn. (D) A higher concentration of Gln in the buffer gave rise to higher concentrations of ammonium. The results shown in panels (B-D) are the average of three independent experiments, with the SDs indicated.

our prediction. This result further indicates that no other amino acid transporter in E. coli can substitute for GadC in mediating Gln-mediated acid resistance.

We sought to restore acid resistance by transforming the $E$. coli variant $\Delta g a d C$ with a plasmid that expresses the $\mathrm{GadC}$ protein. Under acidic conditions where these $E$. coli failed to survive in the presence of Gln, transformation of the $g a d C$-expressing plasmid, but not the empty plasmid, allowed significant survival in the presence of Gln (Figure 4B). Expression of GadC was detected only in $E$. coli cells transformed with the gadC-expressing plasmid, but not the empty plasmid (Supplementary information, Figure S3, lanes 3 and 4). Finally, the concentrations of released ammonium increased rapidly in the gadC-transformed E. coli, but not in the vector control (Figure 4C). Taken together, these results demonstrate that the transporter GadC is required for Gln- and $y b a S$ mediated acid resistance in E. coli.

\section{Sufficiency of YbaS-GadC for acid resistance}

Upon enzymatic deamidation by $\mathrm{YbaS}, \mathrm{Gln}$ is converted to Glu, which can be further decarboxylated by GadA or GadB to generate GABA. The latter reaction, as part of AR2, also contributes to acid resistance. To investigate whether the YbaS-GadC system alone is sufficient to confer acid resistance in $E$. coli, we generated three additional $E$. coli variants, with deletion of $\operatorname{gad} A$, gadB, or gadA and gadB (abbreviated as gadAB), and compared their survival to the WT $E$. coli under the acidic environment of $\mathrm{pH} 2.5$ (Figure 5A). As anticipated, deletion of $\operatorname{gad} A$ or $\operatorname{gadB}$ alone had little impact on the survival of $E$. coli cells in the presence of either $10 \mathrm{mM}$ Glu or $10 \mathrm{mM}$ 
A

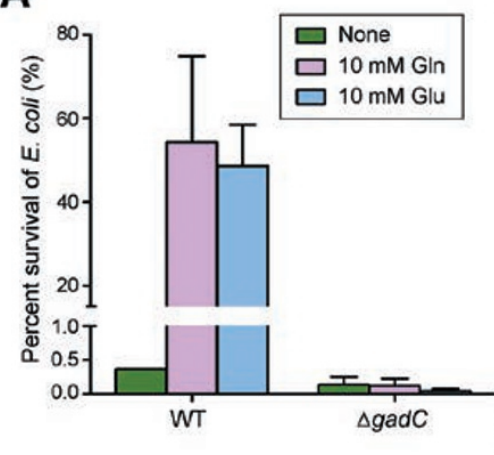

B

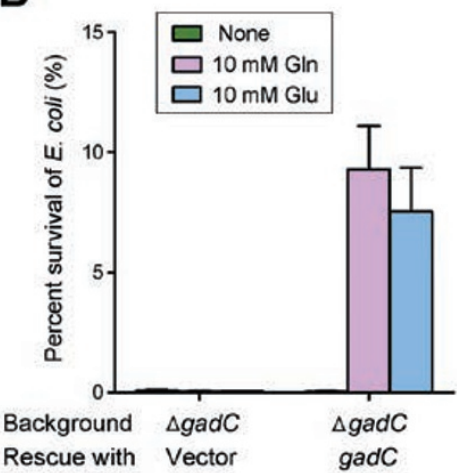

Release of $\mathrm{NH}_{4}^{+}$under $10 \mathrm{mM} \mathrm{Gln}$

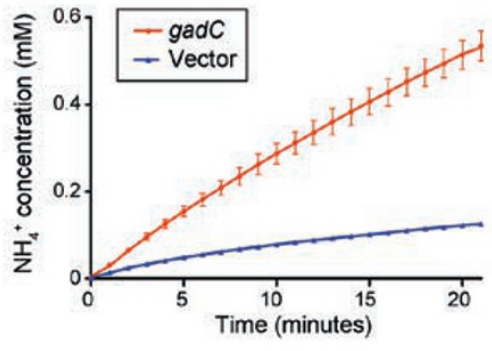

Figure 4 The amino acid antiporter GadC is required for the GIn- and YbaS-mediated acid resistance. (A) Deletion of the gadC gene resulted in loss of acid resistance in the presence of $10 \mathrm{mM}$ Gln. Shown here are results of acid resistance assays performed on WT E. coli and its variant $\triangle$ gadC. (B) Gln-dependent survival of $E$. coli $\Delta$ gadC under acidic environment can be rescued by transformation of a gadC-expressing plasmid. (C) Restoration of ammonium release by transformation of the gadC-expressing plasmid, but not the empty plasmid. The results shown in panels (A-C) represent the average of three independent experiments, with the SDs indicated.

Gln. Simultaneous deletion of $\operatorname{gad} A$ and $\operatorname{gadB}$, however, crippled the ability of $E$. coli to survive in the presence of $10 \mathrm{mM} \mathrm{Glu}$, but not $10 \mathrm{mM}$ Gln. The inability to decarboxylate Glu led to abrogation of acid resistance in this $E$. coli variant in the presence of $10 \mathrm{mM} \mathrm{Glu}$; yet the Gln-mediated acid resistance system, via YbaS and GadC, was still functional. These results indicate that the YbaS-GadC system is sufficient to confer acid resistance in $E$. coli through YbaS-mediated enzymatic deamidation of Gln and GadC-mediated exchange of extracellular Gln with intracellular Glu. Consistent with this conclusion, ambient ammonium concentrations of the $E$. coli variant $\triangle \operatorname{gad} A B$, but not $E$. coli $\triangle y b a S$, increased steadily under acid shock of $\mathrm{pH} 2.5$ in the presence of Gln (Figure 5B).

To further corroborate our conclusion, we sought to detect and quantify the reaction products of Gln in WT $E$. coli and the two variants $\triangle g a d A B$ and $\triangle y b a S$, following incubation of these $E$. coli cells with Gln at $\mathrm{pH} 2.5$. The reaction products from the WT and variant $E$. coli were differentially labeled with a unique reagent TMT, which is specifically designed for mass spectrometry-based quantification through the formation of a covalent adduct with primary amine. Subsequent mass spectrometric (MS) analysis and MS/MS fragmentation allow quantification of each amino acid and its reaction products. Compared to the Gln-alone control, the combined amounts of Glu and Gln remained largely unchanged for the $E$. coli variants $\triangle y b a S$ and $\triangle g a d A B$ (Supplementary information, Figure S6, upper panel). This result is fully consistent with our prediction. In the case of WT E. coli, Gln and Glu together accounted for $\sim 80 \%$ of the input Gln (Supplementary information, Figure S6, upper panel), sug- gesting that roughly $20 \%$ had been converted to reaction products other than Gln or Glu. Supporting this analysis, GABA was readily detectable by MS/MS fragmentation in the reaction products derived from WT E. coli (Supplementary information, Figure S6, middle panel). In the case of $E$. coli variant $\triangle \operatorname{gad} A B$, GABA was undetectable, yet $\sim 25 \%$ of Gln had been converted to Glu (Supplementary information, Figure S6, lower panel). This result nicely corroborates the conclusion that the YbaS-GadC system alone is sufficient to confer acid resistance in $E$. coli. Together, the MS analysis is fully consistent with our genetic and biochemical studies (Figures 2-5).

\section{Discussion}

In this study, we report the identification of a new acid resistance system that relies on the glutaminase YbaS and the amino acid antiporter GadC in E. coli (Figure 5C). Consistent with the need of acid resistance, YbaS (this study) and GadC [15] are activated by acidic $\mathrm{pH}$, and only function properly at $\mathrm{pH} 6.0$ or lower. An essential feature of this AR is the requirement of Gln in the acidic environment. Given the abundance of Gln both in natural environment and in food sources (Supplementary information, Table S1), this new AR is likely to have a key role for bacterial survival.

Our experimental evidence suggests that the YbaSGadC system alone is sufficient for acid resistance, as abrogation of Glu decarboxylation still allowed survival of $E$. coli under pH 2.5 in the presence of Gln (Figure $5 \mathrm{~A})$. We note, however, the percentage of survived $E$. coli variant $\triangle g a d A B$ has decreased by $\sim 50 \%$ from that of 

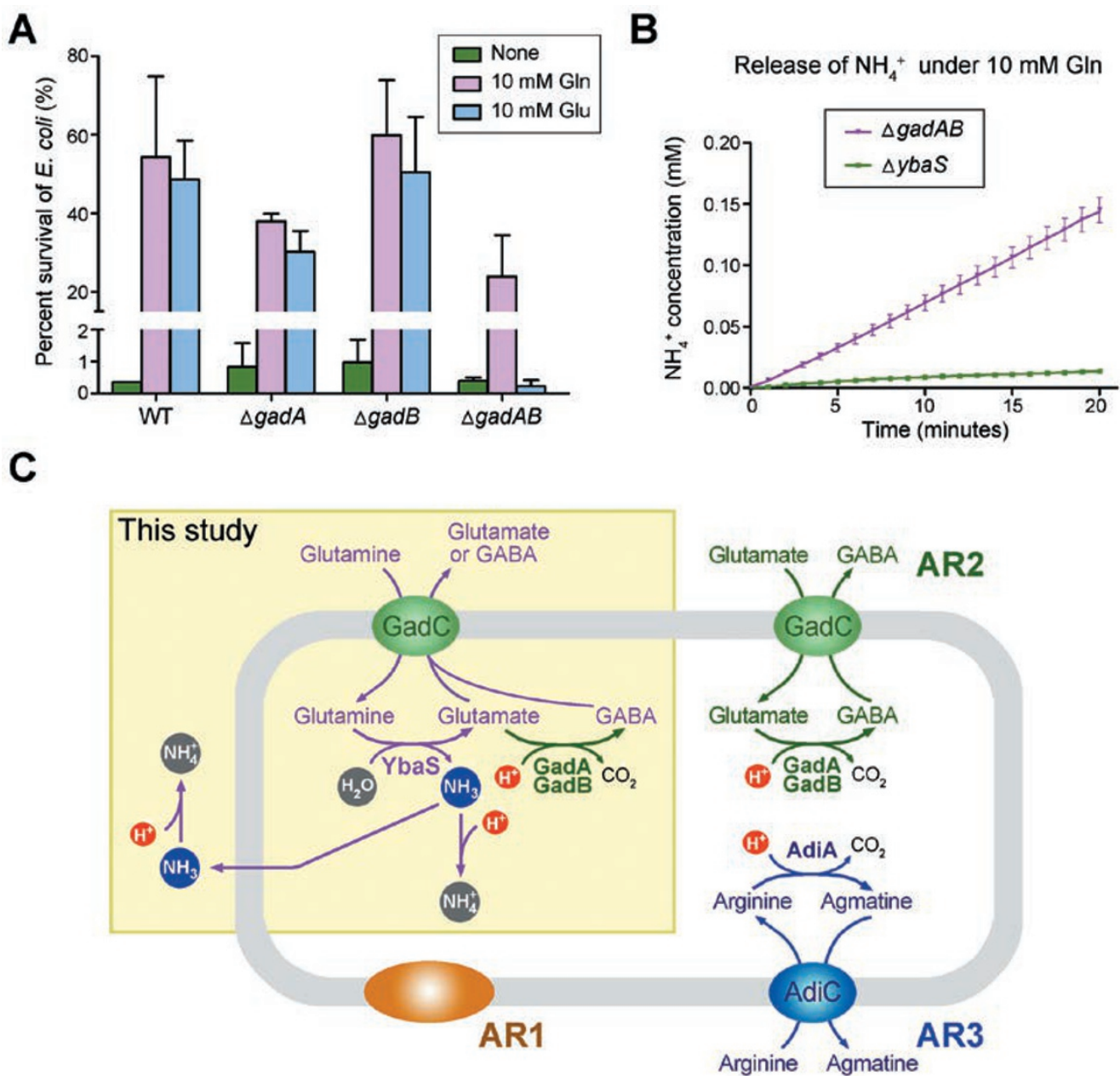

Figure 5 The glutaminase YbaS and the amino acid antiporter GadC together constitute an acid resistance system. (A) Simultaneous deletion of the Glu decarboxlyase genes gadA and gadB still allowed survival of $E$. coli under $\mathrm{pH} 2.5$. Shown here are the results of acid resistance assays performed on $E$. coli of various genetic backgrounds. Deletion of $g a d A$ or gad $B$ alone had little impact on the survival of $E$. coli in the presence of $10 \mathrm{mM}$ Glu or Gln under acidic environment. Deletion of both gadA and gadB resulted in loss of acid resistance in the presence of Glu, but not GIn. (B) Simultaneous deletion of the gad $A$ and gadB genes in $E$. coli still allowed time-dependent accumulation of ammonium in the medium. By contrast, deletion of the ybaS gene abrogated production of ammonium. The results shown in panels (A, B) reflect the average of three independent experiments, with the SDs indicated. (C) A summary of known ARs in E. coli. Although some features of AR1 are known, the full components remain to be identified. Both AR2 and AR3 contain two components. In AR2, GadC exchanges extracellular Glu and intracellular GABA, whereas GadA/GadB converts Glu to GABA by removing the $\alpha$-carboxylate group of Glu. In AR3, AdiC uptakes extracellular Arg and expels intracellular agmatine (Agm), whereas AdiA decarboxylates Arg into Agm. The new AR system discovered by this study also contains two components: GadC and YbaS. GadC is an amino acid antiporter with specificity for GIn, Glu and GABA.

the WT E. coli (Figure 5A). Accordingly, accumulation of the released ammonium ion in the medium was markedly reduced in E. coli $\triangle$ gadAB compared to WT E. coli or E. coli $\Delta y n e H$ (Supplementary information, Figure S7). This analysis argues that the YbaS-GadC system may work best together with a subsequent decarboxylation step by GadA/GadB (Figure 5C).

Survival of any organism, not just bacteria, must rely on its ability to maintain an adequate $\mathrm{pH}$ range. The physiological and biological buffer system is known to rely mainly on ammonia and carbon dioxide [21], which are responsible for neutralization of acid (proton) and base (hydroxide ion), respectively. In this regard, biological reactions that involve adsorption or release of $\mathrm{NH}_{3} / \mathrm{CO}_{2}$ are likely to play an important role in the regulation of acid-base balance in living organisms. For example, decarboxylation of an amino acid, exemplified by the action of GadA/GadB, results in fixation of a pro- 
ton, helping to raise intracellular $\mathrm{pH}$. Although only Arg and Glu are currently known to undergo such reactions in E. coli, there is no reason why other amino acids cannot have a similar role in other bacteria and organisms. Along the same line of logic, enzymatic deamidation of Gln and Asn, which releases $\mathrm{NH}_{3}$, should also confer a means of acid resistance. In this study, we discovered the Gln-mediated acid resistance system. It is tempting to speculate that Asn-related system might function in some other bacteria or organisms. Notably, release of $\mathrm{NH}_{3}$ for acid resistance, as demonstrated in this study for $E$. coli, has been previously demonstrated in the bacterium Helicobacter pylori, which fights gastric acid through enzymatic breakdown of urea by urease [22, 23]. Notably, a variety of bacterial species utilize the arginine deiminase system to help fight acid stress, which converts arginine to ornithine, ammonia and carbon dioxide [24]. Similar mechanisms might be much more widespread than reported to date.

\section{Materials and Methods}

\section{Bacterial strain and culture condition}

Most experiments reported in this manuscript were performed using the E. coli strain MG1655, which has been widely used in the study of acid resistance by $E$. coli $[5,12]$. Deletion of specific gene(s) in this E. coli strain was generated using the one-step gene inactivation technique [25]. To create rescue plasmids, E. coli gadC and $y b a S$ genes were subcloned into the pQLINK vector [26], each with a His6 tag at the N-terminus. The expression levels of GadC and YbaS were monitored by western blot using a monoclonal antibody against the His6 tag. Control experiments were performed with cells transformed with the empty pQLINK vector. In addition, the Gram-negative bacterium $S$. flexneri was purchased from China General Microbiological Culture Collection (CGMCC). The minimal medium used was E medium supplemented with $0.5 \%$ glucose (known as the EG medium [27]). E medium contains 73 $\mathrm{mM} \mathrm{K}_{2} \mathrm{HPO}_{4}, 17 \mathrm{mM} \mathrm{NaNH}_{4} \mathrm{HPO}_{4}, 0.8 \mathrm{mM} \mathrm{MgSO}_{4}$ and $10 \mathrm{mM}$ citrate. The EG medium was adjusted to $\mathrm{pH} 2.5$ using hydrochloric acid $(\mathrm{HCl})$ and sterile filtered. The complex medium used was Luria-Bertani (LB) broth, supplemented with $0.4 \%$ glucose and $100 \mathrm{mM}$ morpholinepropanesulfonic acid buffer (MOPS, $\mathrm{pH}$ 8.0). The complex medium is known as the LBG medium.

\section{Acid resistance assay}

The acid resistance system was assayed after overnight $(20 \mathrm{~h})$ growth in the LBG medium. The overnight culture was diluted

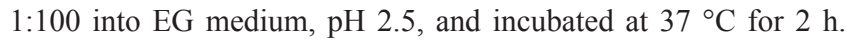
Then the cells were diluted in LB medium, plated to LB agar, and incubated at $37^{\circ} \mathrm{C}$ for $15 \mathrm{~h}$ before colony count. The numbers of colony serve as the numerators for calculation of percent $E$. coli survival. In the mean time, the overnight culture without acid shock was diluted in LB medium, plated on LB agar, and incubated at $37^{\circ} \mathrm{C}$ for $15 \mathrm{~h}$; the number of colony serves as the denominator for calculation of percent $E$. coli survival. The $E$. coli cell densities of the overnight culture in different experiments were approximately the same at $5 \times 10 \% / \mathrm{ml}$. Survival values reported are the averages of three independent experiments.

To assay the role of individual amino acid, overnight $E$. coli culture was diluted 1:100 into EG medium, $\mathrm{pH} 2.5$, supplemented with one of the 20 natural L-amino acids at $1 \mathrm{mM}$. The negative control for these survival experiments involved an acid challenge (pH 2.5) in EG medium without amino acid supplement.

\section{Protein preparation}

The cDNAs of the full-length $\operatorname{gadA}, \operatorname{gadB}$, yneH and $y b a S$ genes were individually cloned into pET15b (Novagen). Overexpression of the recombinant protein was induced by $0.2 \mathrm{mM}$ isopropyl $\beta$-d-thiogalactoside (IPTG) when the cells reached an optical density value of 1.2 at $600 \mathrm{~nm}$. After growth at $18{ }^{\circ} \mathrm{C}$ for $12 \mathrm{~h}$, the cells were collected, homogenized in a buffer containing $25 \mathrm{mM}$ Tris- $\mathrm{HCl}, \mathrm{pH} 8.0$, and $150 \mathrm{mM} \mathrm{NaCl}$. After sonication, cell debris was removed by low-speed centrifugation for $1 \mathrm{~h}$. The supernatant was collected and loaded onto $\mathrm{Ni}^{2+}$-nitrilotriacetate affinity resin (Ni-NTA, Qiagen), followed by a wash with $25 \mathrm{mM}$ Tris- $\mathrm{HCl}, \mathrm{pH} 8.0,150 \mathrm{mM} \mathrm{NaCl}$ and $20 \mathrm{mM}$ imidazole. The target protein was eluted by an elution buffer containing $250 \mathrm{mM}$ imidazole, $25 \mathrm{mM}$ Tris, $\mathrm{pH} 8.0$ and $150 \mathrm{mM} \mathrm{NaCl}$, and further purified by anion exchange chromatography (Source-15Q, GE Healthcare). The protein was further purified by gel filtration (Superdex-200, $10 / 30$, GE Healthcare) with a buffer containing $150 \mathrm{mM} \mathrm{NaCl}$ and $25 \mathrm{mM}$ Tris, $\mathrm{pH}$ 8.0.

\section{Glutaminase activity assay}

The initiation solution, containing $0.1 \mathrm{ml}$ of freshly made Gln solution at desired concentration, $10 \mu \mathrm{l}$ of $1 \mathrm{M}$ buffer solution and $37.5 \mu 1 \mathrm{H}_{2} \mathrm{O}$, was equilibrated at $37{ }^{\circ} \mathrm{C}$. The reaction was started by adding $2.5 \mu \mathrm{l}$ of $0.1 \mathrm{mg} / \mathrm{ml}$ protein ( $\mathrm{YbaS}$ or $\mathrm{YneH})$ to the initiation solution and allowed to continue at $37^{\circ} \mathrm{C}$ for $1 \mathrm{~min}$. The reaction was stopped by boiling for $10 \mathrm{~min}$. A $50-\mu 1$ aliquot of the supernatant was added to a reaction mixture containing $0.5 \mathrm{ml}$ of hydroxylamine buffer ( $0.25 \mathrm{M}$ hydroxylamine and $20 \mathrm{mM}$ EDTA, $\mathrm{pH} 8.0), 25 \mu \mathrm{l}$ of $100 \mathrm{mM} \mathrm{NAD}^{+}$solution, $0.425 \mathrm{ml}$ of distilled water and 5 units of bovine Glu dehydrogenase (Sigma-Aldrich). After incubation at $37{ }^{\circ} \mathrm{C}$ for $1 \mathrm{~h}$, the optical density at $340 \mathrm{~nm}$ was measured. For determination of $V_{\max }$ and $K_{\mathrm{m}}$ at different $\mathrm{pH}$ values, six serial dilutions of Gln were made to achieve a range of concentrations from $50 \mathrm{mM}$ to $1.67 \mathrm{mM}$. Measurements were done in triplicates and analyzed using GRAPHPAD PRISM 5.0 Demo (GraphPad Software, San Diego, CA, USA).

\section{Decarboxylation reaction for $M S$ analysis}

Solution A contained $10 \mu \mathrm{g}$ GadA or GadB, $10 \mathrm{mM}$ citrate, $\mathrm{pH} 4.5$, and $0.5 \mathrm{mM}$ pyridoxal 5'-phosphate (PLP). Solution B contained $20 \mathrm{mM}$ Glu or Gln, $10 \mathrm{mM}$ citrate, $\mathrm{pH} 4.5$, and $0.5 \mathrm{mM}$ PLP. Both solutions A and B were individually equilibrated at 37 ${ }^{\circ} \mathrm{C}$. The reaction was started by mixing $100 \mu 1$ solution A with 100 $\mu 1$ solution B and allowed to proceed at $37^{\circ} \mathrm{C}$ for $10 \mathrm{~min}$. Reaction was stopped by flash-freeze with liquid nitrogen, and samples were subjected to MS detection.

\section{Ammonium release assay}

The acid resistance system was assayed after overnight $(20 \mathrm{~h})$ growth in LB broth buffered to $\mathrm{pH} 8.0$ with $100 \mathrm{mM}$ MOPS and supplemented with $0.4 \%$ glucose (LBG medium). The initial cell 
density was measured, and adjusted to the same $\mathrm{OD}_{600}$ value. One $\mathrm{ml}$ of cell culture was centrifuged. The pellet was washed with distilled water, pelleted again and resuspended in distilled water

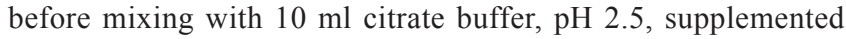
with a specific amino acid. The ammonium concentration was continuously measured by the DUAL STAR Ammonium Ion Selective Electrode Meter (Thermo Scientific Orion).

\section{Mass spectrometry}

For detection and quantification of Gln/Gln and their enzymatic reaction products, samples were labeled with a series of TMT reagents (Thermo, Pierce Biotechnology, Rockford, IL, USA) according to the manufacturer's instruction. Each TMT reagent is an isobaric tag, comprising an amine-reactive NHS-ester group, a spacer arm, and an MS/MS reporter [28, 29]. Each sample is labeled with a unique TMT reagent, which gives rise to a unique reporter mass during fragmentation in the MS/MS spectrum. These reporter ions, in the low mass region of the MS/MS spectrum, are used to report relative concentrations of the target molecules in distinct samples. Each TMT reagent, out of a set of six reagents in total, has the same nominal parent (precursor) mass, and the labeled molecules from different samples also have the same mass; however, the masses differ in the balancing and reporter region, and are detected in the MS/MS spectra.

Briefly, the TMT reagents were dissolved by anhydrous acetonitrile. The labeling reaction was carried out by incubation of Gln/ Glu and their derivatives with the TMT reagents for $1 \mathrm{~h}$ at room temperature, and the reaction was quenched using hydroxylamine. The TMT-labeled Gln/Glu and their derivatives were analyzed by LC-MS/MS. The TMT-labeled products were separated by a $65-$ min gradient elution at a flow rate $0.25 \mu \mathrm{l} / \mathrm{min}$ with the EASYnLCII- integrated nano-HPLC system (Proxeon, Denmark), which is directly interfaced with the Thermo LTQ-Orbitrap mass spectrometer. The analytical column was a home-made fused silica capillary column (75 $\mu \mathrm{m}$ ID, $150 \mathrm{~mm}$ length; Upchurch, Oak Harbor, WA, USA) packed with C-18 resin (300 A, $5 \mu \mathrm{m}$, Varian, Lexington, MA, USA). Mobile phase A consisted of $0.1 \%$ formic acid, and mobile phase B consisted of $100 \%$ acetonitrile and $0.1 \%$ formic acid. The LTQ-Orbitrap mass spectrometer was operated in the data-dependent acquisition mode using the Xcalibur 2.0.7 software, and there is a single full-scan mass spectrum in the Orbitrap (100-1 $000 \mathrm{~m} / \mathrm{z}, 30000$ resolution) followed by three MS/ MS scans in the quadruple collision cell using the higher energy collision dissociation (HCD), in which the precursor mass selection window was set as $2 \mathrm{Da}$. The MS/MS spectra from each LCMS/MS run were analyzed to find the labeled Gln/Glu and their corresponding fragments.

\section{Acknowledgments}

This work was supported by funds from the Ministry of Science and Technology (2009CB918801), the National Natural Science Foundation of China (31130002, 31021002, and 30888001), and the Beijing Municipal Commissions of Education and Science and Technology.

\section{References}

1 Riley LW, Remis RS, Helgerson SD, et al. Hemorrhagic colitis associated with a rare Escherichia coli serotype. $N$ Engl J Med 1983; 308:681-685.

2 Rohde H, Qin J, Cui Y, et al. Open-source genomic analysis of Shiga-toxin-producing E. coli O104:H4. N Engl J Med 2011; 365:718-724.

3 Foster JW. Escherichia coli acid resistance: tales of an amateur acidophile. Nat Rev Microbiol 2004; 2:898-907.

4 Lin J, Lee IS, Frey J, Slonczewski JL, Foster JW. Comparative analysis of extreme acid survival in Salmonella typhimurium, Shigella flexneri, and Escherichia coli. J Bacteriol 1995; 177:4097-4104.

5 Lin J, Smith MP, Chapin KC, Baik HS, Bennett GN, Foster JW. Mechanisms of acid resistance in enterohemorrhagic Escherichia coli. Appl Environ Microbiol 1996; 62:3094-3100.

6 Castanie-Cornet MP, Foster JW. Escherichia coli acid resistance: cAMP receptor protein and a $20 \mathrm{bp}$ cis-acting sequence control $\mathrm{pH}$ and stationary phase expression of the gadA and gadBC glutamate decarboxylase genes. Microbiology 2001; 147:709-715.

7 Castanie-Cornet MP, Penfound TA, Smith D, Elliott JF, Foster JW. Control of acid resistance in Escherichia coli. J Bacteriol 1999; 181:3525-3535.

8 Hersh BM, Farooq FT, Barstad DN, Blankenhorn DL, Slonczewski JL. A glutamate-dependent acid resistance gene in Escherichia coli. J Bacteriol 1996; 178:3978-3981.

9 Waterman SR, Small PL. Identification of sigma S-dependent genes associated with the stationary-phase acid-resistance phenotype of Shigella flexneri. Mol Microbiol 1996; 21:925-940.

10 Smith DK, Kassam T, Singh B, Elliott JF. Escherichia coli has two homologous glutamate decarboxylase genes that map to distinct loci. J Bacteriol 1992; 174:5820-5826.

11 De Biase D, Tramonti A, John RA, Bossa F. Isolation, overexpression, and biochemical characterization of the two isoforms of glutamic acid decarboxylase from Escherichia coli. Protein Expr Purif 1996; 8:430-438.

12 Iyer R, Williams C, Miller C. Arginine-agmatine antiporter in extreme acid resistance in Escherichia coli. J Bacteriol 2003; 185:6556-6561.

13 Gong S, Richard H, Foster JW. YjdE (AdiC) is the arginine: agmatine antiporter essential for arginine-dependent acid resistance in Escherichia coli. J Bacteriol 2003; 185:4402-4409.

14 Stim KP, Bennett GN. Nucleotide sequence of the adi gene, which encodes the biodegradative acid-induced arginine decarboxylase of Escherichia coli. J Bacteriol 1993; 175:12211234.

15 Ma D, Lu P, Yan C, et al. Structure and mechanism of a glutamate-GABA antiporter. Nature 2012; 483:632-636.

16 Richard H, Foster JW. Escherichia coli glutamate- and arginine-dependent acid resistance systems increase internal $\mathrm{pH}$ and reverse transmembrane potential. J Bacteriol 2004; 186:6032-6041.

17 Brown G, Singer A, Proudfoot M, et al. Functional and structural characterization of four glutaminases from Escherichia coli and Bacillus subtilis. Biochemistry 2008; 47:5724-5735.

18 Prusiner S, Davis JN, Stadtman ER. Regulation of glutaminase B in Escherichia coli. I. Purification, properties, and cold lability. J Biol Chem 1976; 251:3447-3456.

19 Hartman SC. Glutaminase of Escherichia coli. I. Purification and general catalytic properties. J Biol Chem 1968; 243:853- 
863.

20 Khademi S, O'Connell J III, Remis J, Robles-Colmenares Y, Miercke LJ, Stroud RM. Mechanism of ammonia transport by Amt/MEP/Rh: structure of AmtB at 1.35 A. Science 2004; 305:1587-1594.

21 Krulwich TA, Sachs G, Padan E. Molecular aspects of bacterial pH sensing and homeostasis. Nat Rev Microbiol 2011; 9:330-343.

22 Stingl K, De Reuse H. Staying alive overdosed: how does Helicobacter pylori control urease activity? Int J Med Microbiol 2005; 295:307-315.

23 Pflock M, Kennard S, Finsterer N, Beier D. Acid-responsive gene regulation in the human pathogen Helicobacter pylori. $J$ Biotechnol 2006; 126:52-60.

24 Marquis RE, Bender GR, Murray DR, Wong A. Arginine deiminase system and bacterial adaptation to acid environments. Appl Environ Microbiol 1987; 53:198-200.

25 Datsenko KA, Wanner BL. One-step inactivation of chromosomal genes in Escherichia coli K-12 using PCR products. Proc Natl Acad Sci USA 2000; 97:6640-6645.

26 Scheich C, Kummel D, Soumailakakis D, Heinemann U, Bus- sow K. Vectors for co-expression of an unrestricted number of proteins. Nucleic Acids Res 2007; 35:e43.

27 Vogel HJ, Bonner DM. Acetylornithinase of Escherichia coli: partial purification and some properties. J Biol Chem 1956; 218:97-106.

28 Thompson A, Schafer J, Kuhn K, et al. Tandem mass tags: a novel quantification strategy for comparative analysis of complex protein mixtures by MS/MS. Anal Chem 2003; 75:18951904.

29 Dayon L, Hainard A, Licker V, et al. Relative quantification of proteins in human cerebrospinal fluids by MS/MS using 6-plex isobaric tags. Anal Chem 2008; 80:2921-2931.

(Supplementary information is linked to the online version of the paper on the Cell Research website.)

This work is licensed under the Creative Commons Attribution-NonCommercial-No Derivative Works 3.0 Unported License. To view a copy of this license, visit http:// creativecommons.org/licenses/by-nc-nd/3.0 